\title{
Can tumor mutational burden determine the most effective treatment for lung cancer patients?
}

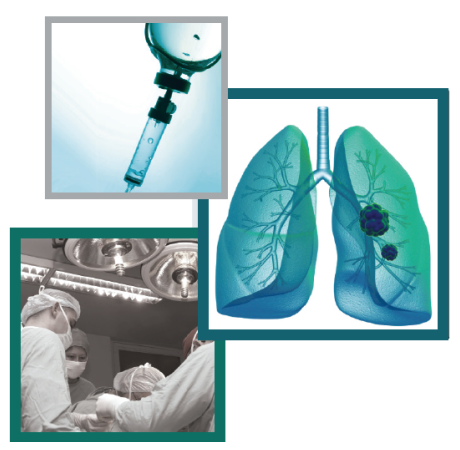

Shixiang Wang ${ }^{1}$, Zaoke $\mathrm{He}^{1}$, Xuan Wang ${ }^{1}$, Huimin $\mathrm{Li}^{1}$, Tao $\mathrm{Wu}^{1}$, Xiaoqin Sun ${ }^{1}, \mathrm{Kai} \mathrm{Wu}^{2}$

\& Xue-Song Liu*,1(i)

${ }^{1}$ School of Life Science \& Technology, ShanghaiTech University, Shanghai 201203, China

${ }^{2}$ Department of Thoracic Surgery, the First Affiliated Hospital of Zhengzhou University, Zhengzhou 450052, China

*Author for correspondence: liuxs@shanghaiTech.edu.cn

"These studies have led to the first tissue-agnostic approval for anti-PD-1 therapy across unresectable or metastatic solid tumors with microsatellite instability or mismatch repair deficiency"

\section{Graphical abstract:}

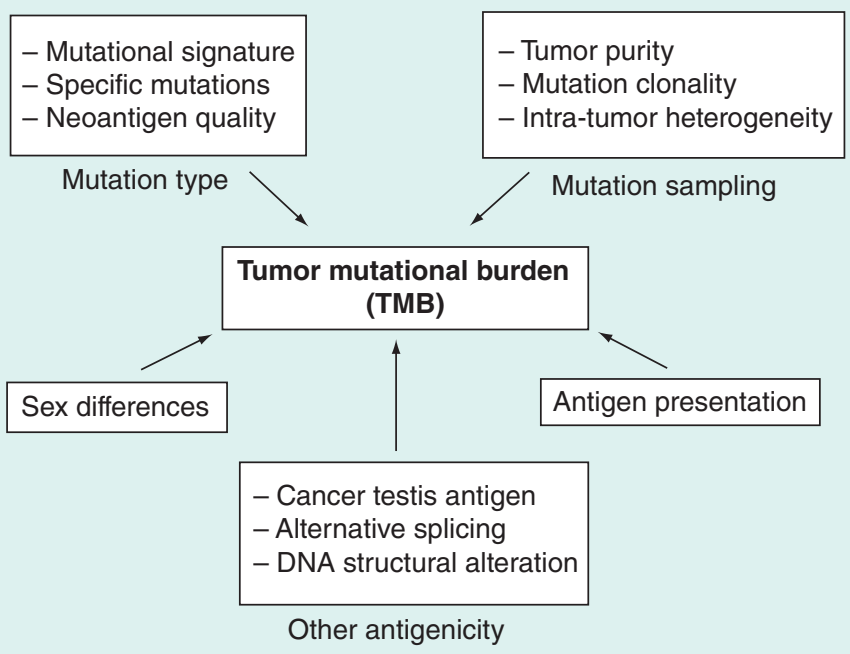

First draft submitted: 8 December 2019; Accepted for publication: 17 December 2019; Published online: 14 January 2020

Keywords: biomarker $\bullet$ cancer immunotherapy $\bullet$ immune checkpoint inhibitor $\bullet$ lung cancer $\bullet$ TMB $\bullet$ tumor mutational burden

Immunotherapy has revolutionized the treatment of cancer, and this success is represented by immune checkpoint inhibitors (ICI) (including anti-PD-1 antibodies, anti-PD-L1 antibodies, anti-CTLA- 4 antibodies and their combinations), which show higher efficacy than standard therapies in several cancers, including lung cancer. However, the majority of patients do not respond to immunotherapy, and biomarkers for predicting immunotherapy clinical response are urgently needed. Most tumor types show response rates below $40 \%$ to PD-1 inhibition and the objective response rates of each tumor type are reported to be highly correlated with the tumor mutational burden (TMB) of each tumor type [1]. In addition to TMB, multiple other factors are reported to affect ICI effectiveness, 
including $P D-L 1$ expression, the degree of cytotoxic T-cell infiltration, mutational signature, antigen presentation defects, interferon signaling, tumor aneuploidy, T-cell gene expression signatures and microbiota [2].

The initial evidence supporting the correlation between high nonsynonymous mutational burden and improved clinical benefit obtained with immunotherapy was demonstrated by using whole-exome sequencing in advanced non-small-cell lung cancer treated with anti-PD-1 antibody and their matched normal DNA [3]. Later clinical trials have confirmed high TMB correlates with enhanced immunotherapy responses in other cancer types, such as head and neck cancer and urothelial carcinoma [4]. Tumors with DNA mismatch repair deficiency can display high microsatellite instability and accumulate substantial numbers of somatic mutations. A high objective response rate has been demonstrated across mismatch repair-deficient tumors to anti-PD-1 therapy [5,6]. These studies have led to the first tissue-agnostic approval for anti-PD-1 therapy across unresectable or metastatic solid tumors with microsatellite instability or mismatch repair deficiency.

Originally, TMB was detected with whole-exome sequencing, while recently, targeted gene panel sequencing has been widely used in clinics for TMB assessment. There is no consensus definition of TMB, different clinical practices use different definitions with different detecting methods. Foundation Medicine (MA, USA), defined $\mathrm{TMB}$ as the number of base substitutions (including synonymous mutations) in the coding region of targeted genes. Germline DNA was not sequenced but filtering for both oncogenic driver alterations and germline variants was carried out using public databases. The Memorial Sloan Kettering Cancer Center (NY, USA) approach quantified nonsynonymous mutations using sequencing data from both tumor and germline DNA. Several other different target gene panels have also been reported and used in clinics [7].

Targeted gene panel sequencing with formalin-fixed and paraffin-embedded samples appears to be a more feasible and straight-forward approach for TMB assessment in clinics. However, formalin fixative is known to induce various crosslinks, which are the main source of sequencing artifacts, notably through DNA fragmentation, denaturation and cytosine deamination. Blood TMB (bTMB) was assessed using cell-free DNA from blood, bTMB has recently emerged as an effective predictive biomarker for ICI response prediction [8]. For accurate bTMB quantification, mutations derived from clonal hematopoiesis of white blood cells should be well controlled [9]. The TMB cut-off values associated with improved survival varied markedly between cancer types, and there may not be one universal definition of high TMB [10].

Previously, it has been reported that TMB shows imperfect correlation with ICI response in that mutation load distributions overlap considerably between responders and nonresponders [11]. In addition, TMB does not correlate with the immunotherapy clinical response in some tumor types, including Hodgkin's lymphoma and renal cell carcinoma [4]. Furthermore, no clinical study has confirmed an overall survival advantage in high-TMB patients compared with low-TMB patients after immunotherapy. As reviewed recently, TMB also has some inherent technical issues that could dampen its clinical utility $[12,13]$. Here, we summarize that the following factors should be carefully considered for further improvement of TMB-based immunotherapy biomarkers.

\section{Mutation type}

Mutations are not the same, and some types of DNA mutations can be more efficient in ICI response prediction compared with others. For example, APOBEC mutation signature has been reported to predict immunotherapy response more effectively than total TMB [14]. Different types of mutations could have different effects on the coding peptide, which could lead to differences in peptide hydrophobicity and/or immunogenicity. Neoantigen quality has been proposed to assess the immunogenicity of neoantigens, and the overall quality of neoantigens should replace simple TMB in future immunotherapy response prediction. In addition, some specific mutation itself can affect immunotherapy response, for example, JAK1, JAK2, b2M, SKT11, SERPINB3 and SERPINB4 mutations [15,16]. The rational inclusion of these mutations in TMB quantification needs to be carefully designed.

\section{Other tumor antigenicity}

Cancer germline (also known as cancer testis) antigens are normally expressed in germ cells and trophoblast tissues and are aberrantly expressed in a variety of human malignancies. Cancer germline antigens are important sources for tumor antigenicity, and this antigenicity should also be considered for future improvement of TMB. Specific alternative splicing in cancer cells can encode for a de novo protein, which is not expressed in normal tissues, and this novel protein can evoke an immune response as a tumor antigen. DNA structural alterations and so called 'noncoding' regions could also encode for a novel tumor specific peptide/protein, and these types of tumor antigenicity should also be considered in future biomarker design. 


\section{Mutation sampling}

Clonal and subclonal TMB can have different effects on immunotherapy response prediction [17]. Due to intratumor heterogeneity, different regions of the tumor could have different mutational burdens. This regional effect should be carefully considered, especially for primarily localized cancer. Low tumor purity can also influence mutation calling, and consequently influence TMB assessment. For clinical practice, especially tissue TMB detection, minimum tumor purity level is required.

\section{Sex differences}

Recently, Wang et al. reported that the predictive power of TMB in lung cancer immunotherapy response is influenced by patient's sex, and for male lung cancer patients, TMB is a relatively poor biomarker in immunotherapy response prediction [18]. This sex difference in TMB's predictive performance is probably caused by the enhanced immune response in females compared with males [19]. Future development of immunotherapy biomarkers should consider sex differences and special efforts should be paid to improve the performance of immunotherapy predictive biomarkers for male lung cancer patients.

\section{Antigen presentation status}

TMB reflects tumor antigenicity. To evoke an immune response, this tumor antigenicity should be presented to the immune cells for reorganization and specific killing. Recently, antigen presentation gene expression signature has been combined with TMB to generate a novel biomarker, tumor immunogenicity score. In both correlation with pan-cancer ICI objective response rates and ICI clinical response prediction for individual patients, tumor immunogenicity score consistently showed improved performance compared with TMB and other known prediction biomarkers for ICI response [20].

In conclusion, immunotherapy revolutionized the treatment of lung cancer, and has demonstrated success in some originally incurable late-state lung cancer. TMB is a widely used clinical biomarker for ICI response prediction; however, TMB itself is not sufficient for the selection of lung cancer patients for immunotherapy. Several factors need to be considered for the future improvement of TMB-based biomarker, including: mutation type, mutation sampling, other tumor antigenicity, sex differences and antigen presentation status. With all the above factors considered, we may generate a robust predictive biomarker for ICI in lung cancer.

Financial \& competing interests disclosure

This work was supported in part by The National Natural Science Foundation of China (31771373), and by startup funding from ShanghaiTech University. The authors declare that they have no competing interests. The authors have no other relevant affiliations or financial involvement with any organization or entity with a financial interest in or financial conflict with the subject matter or materials discussed in the manuscript apart from those disclosed.

No writing assistance was utilized in the production of this manuscript.

\section{Open access}

This work is licensed under the Attribution-NonCommercial-NoDerivatives 4.0 Unported License. To view a copy of this license, visit http://creativecommons.org/licenses/by-nc-nd/4.0/

\section{References}

Papers of special note have been highlighted as: $\bullet$ of interest $\bullet \bullet$ of considerable interest

1. Yarchoan M, Hopkins A, Jaffee EM. Tumor mutational burden and response rate to PD-1 inhibition. N. Engl. J. Med. 377(25), 2500-2501 (2017).

2. Havel JJ, Chowell D, Chan TA. The evolving landscape of biomarkers for checkpoint inhibitor immunotherapy. Nat. Rev. Cancer 19(3), 133-150 (2019).

3. Rizvi NA, Hellmann MD, Snyder A et al. Cancer immunology. Mutational landscape determines sensitivity to PD-1 blockade in non-small cell lung cancer. Science 348(6230), 124-128 (2015).

4. Keenan TE, Burke KP, Van Allen EM. Genomic correlates of response to immune checkpoint blockade. Nat. Med. 25(3), 389-402 (2019).

- An comprehensive review about genomic biomarkers for immune checkpoint inhibitors.

5. Le DT, Uram JN, Wang H et al. PD-1 blockade in tumors with mismatch-repair deficiency. N. Engl. J. Med. 372(26), 2509-2520 (2015). 
6. Le DT, Durham JN, Smith KN et al. Mismatch repair deficiency predicts response of solid tumors to PD-1 blockade. Science 357(6349), 409-413 (2017).

7. Chan TA, Yarchoan M, Jaffee E et al. Development of tumor mutation burden as an immunotherapy biomarker: utility for the oncology clinic. Ann. Oncol. 30(1), 44-56 (2019).

-. A comprehensive review about the history and the current status for tumor mutational burden.

8. Gandara DR, Paul SM, Kowanetz M et al. Blood-based tumor mutational burden as a predictor of clinical benefit in non-small-cell lung cancer patients treated with atezolizumab. Nat. Med. 24(9), 1441-1448 (2018).

9. Razavi P, Li BT, Brown DN et al. High-intensity sequencing reveals the sources of plasma circulating cell-free DNA variants. Nat. Med. 25, 1928-1937 (2019).

10. Samstein RM, Lee CH, Shoushtari AN et al. Tumor mutational load predicts survival after immunotherapy across multiple cancer types. Nat. Genet. 51(2), 202-206 (2019).

11. Van Allen EM, Miao D, Schilling B et al. Genomic correlates of response to CTLA-4 blockade in metastatic melanoma. Science 350(6257), 207-211 (2015).

12. Addeo A, Banna GL, Weiss GJ. Tumor mutation burden-from hopes to doubts. JAMA Oncol. 5(7), 934-935 (2019).

13. Friedlaender A, Bauml J, Banna GL, Addeo A. Identifying successful biomarkers for patients with non-small-cell lung cancer. Lung Cancer Manag. 8(3), LMT17 (2019).

14. Wang S, Jia M, He Z, Liu XS. APOBEC3B and APOBEC mutational signature as potential predictive markers for immunotherapy response in non-small cell lung cancer. Oncogene 37(29), 3924-3936 (2018).

15. Zaretsky JM, Garcia-Diaz A, Shin DS et al. Mutations associated with acquired resistance to PD-1 blockade in melanoma. N. Engl. J. Med. 375(9), 819-829 (2016).

16. Riaz N, Havel JJ, Kendall SM et al. Recurrent SERPINB3 and SERPINB4 mutations in patients who respond to anti-CTLA4 immunotherapy. Nat. Genet. 48(11), 1327-1329 (2016).

17. Mcgranahan N, Furness AJ, Rosenthal R et al. Clonal neoantigens elicit $\mathrm{T}$ cell immunoreactivity and sensitivity to immune checkpoint blockade. Science 351(6280), 1463-1469 (2016).

18. Wang S, Zhang J, He Z, Wu K, Liu XS. The predictive power of tumor mutational burden in lung cancer immunotherapy response is influenced by patients' sex. Int. J. Cancer 145(10), 2840-2849 (2019).

- Reports for the first time that the predictive power of tumor mutational burden is influenced by patients' sex.

19. Wang S, Cowley LA, Liu XS. Sex differences in cancer immunotherapy efficacy, biomarkers, and therapeutic strategy. Molecules 24(18), doi: 10.3390/molecules24183214 (2019).

20. Wang S, He Z, Wang X, Li H, Liu XS. Antigen presentation and tumor immunogenicity in cancer immunotherapy response prediction. Elife doi:10.7554/eLife.49020 8 (2019). 